\title{
Antioxidant activity of phenolic compounds identified in sunflower seeds
}

\author{
Magdalena Karamać • Agnieszka Kosińska • \\ Isabel Estrella $\cdot$ Teresa Hernández • \\ Montserrat Dueñas
}

Received: 24 February 2012/Revised: 25 April 2012 / Accepted: 2 May 2012/Published online: 22 May 2012

(C) The Author(s) 2012. This article is published with open access at Springerlink.com

\begin{abstract}
The antioxidant activity and phenolic compound profiles of six fractions (I-VI) obtained from sunflower seed extract were studied. HPLC-MS(ESI) analysis was applied for quantitative and qualitative determination of phenolic compounds of the fractions. The antioxidant activity of the fractions was studied in terms of their ability to scavenge DPPH and $\mathrm{ABTS}^{+}$and to reduce $\mathrm{Fe}^{3+} /$ ferricyanide complex to the ferrous form and was expressed as $\mathrm{EC}_{50}$, TEAC and reducing power values, respectively. The results of all antioxidant activity tests showed good correlations among each other and with the phenolic contents for the individual fractions. The fractions IV-VI were characterized by high antioxidant activity. 5-O-Caffeoylquinic acid was a predominant compound of fractions IV and $\mathrm{V}$, while dicaffeoylquinic acid isomers and caffeoyldimethoxycinnamoylquinic acid isomers accounted for $76.6 \%$ of phenolic compounds of fraction VI. Ferulic acid, $p$-coumaroylquinic acid isomers, ferulic acid dehydrotrimer isomers and some quercetin derivatives were also
\end{abstract}

M. Karamać $(\bowtie) \cdot$ A. Kosińska

Division of Food Sciences, Institute of Animal Reproduction and Food Research, Polish Academy of Sciences,

Tuwima 10, 10-748 Olsztyn, Poland

e-mail: m.karamac@pan.olsztyn.pl

I. Estrella $\cdot$ T. Hernández

Instituto de Ciencia y Tecnología de Alimentos y Nutrición,

CSIC, Juan de la Cierva 3, 28006 Madrid, Spain

M. Dueñas

Polyphenols Research Group, Unidad de Nutrición y

Bromatología, Facultad de Farmacia, Universidad de Salamanca,

Campus Miguel de Unamuno, 37007 Salamanca, Spain identified. The highest content of those compounds was noted in fraction III.

Keywords Sunflower seeds - Phenolic compound profile · Antioxidant activity $\cdot$ Sephadex LH-20 column

chromatography $\cdot$ HPLC-MS(ESI)

\section{Introduction}

Antioxidants are applied in food industry as additives limiting oxidation of food components, especially lipids. The oxidation leads to losses in food quality and shelf life [1]. The antioxidants play an important role also in living organisms since they prevent excessive free radical formation in cells. The extent of free radicals may cause disruption of biologically imported molecules, and in consequence the onset of various diseases. It is believed that oxidative processes are among the reasons for deficiency of immunological system and increased risk of infectious diseases, cancer development, diabetes, arthritis, rheumatoid diseases, respiratory diseases, cataracts, atherosclerosis and a series of destructive processes due to aging, schizophrenia and Alzheimer's disease [2].

Fruits and vegetables are important sources of antioxidants. Among dietary plant products, sunflower seeds are characterized by high antioxidant potential [3, 4]. Sunflower is an important oleaginous plant grown in many European countries. The antioxidant compounds are present in both sunflower oil and oilseed-extracted meal [5]. Extracts with high antioxidant activity may also be obtained from sunflower seed shells $[6,7]$. Compared to vegetables oils, sunflower oil is especially rich in $\alpha$-tocopherol [5]. The antioxidant potential of defatted sunflower meal and sunflower seed shells is determined 
mainly by the content of phenolic compounds [5, 6]. However, it has to be mentioned that shells contain only $0.7-5.4 \%$ of sunflower seed phenolics, whereas the rest is located in kernels [8]. It has been estimated that sunflower cake, by-product of oil extraction, contains as much as $2-4 \%$ of phenolic compounds $[9,10]$. For this reason, meal prepared from press residues might become a valuable source of antioxidants for application in food technology. Up to date, sunflower by-product has been primarily used as ruminant feed due to its high protein content [11].

One of the chlorogenic acid isomers-5-O-caffeoylquinic acid-is the predominant phenolic compound in sunflower kernel $[9,12]$. This compound comprises 43-73\% of phenolic compounds extracted from kernels $[8,10]$. The attempts to identify the other phenolic compounds present in sunflower seeds were made in the 1970s [9]; however, the techniques used at that time did not allow the identification of those compounds. The most commonly used approach involved liberation of individual compounds from ester and glycosidic bonds and then their analysis [13]. The identification of native forms of phenolic compounds present in sunflower seeds became possible in the recent years, especially after popularization of LC/MS methods $[8,10,12]$. Until now, researchers using this technique analyzed sunflower seed phenolic compounds directly in aqueous methanolic, aqueous ethanolic or acetonitrile crude extract. Our study aimed to obtain fractions of phenolic compounds from the extract of dehulled sunflower seeds using Sephadex LH-20 column chromatography, to identify phenolic compounds in the fractions and to determine their antioxidant activity.

\section{Materials and methods}

Material and chemicals

Sunflower seeds (Helianthus annuиs) of non-oil type served as a plant material for extraction of phenolic compounds. Dehulled sunflower seeds (sunflower kernels) were purchased in a specialized local shop in Olsztyn (Poland).

All chemicals used were of analytical or HPLC grade. Sephadex LH-20, 1,1-diphenyl-2-picrylhydrazyl radical (DPPH), [2,2'-azinobis-(3-ethylbenzothiazoline-6-sulfonic acid)] diammonium salt (ABTS), 6-hydroxy-2,5,7,8-tetramethyl-chroman-2-carboxylic acid (Trolox) and standards such as $(+)$-catechin and 5- $O$-caffeoylquinic acid were obtained from Sigma-Aldrich (Poznan, Poland). transFerulic acid and quercetin rutinoside were from Extrasynthese (Genay, France), and quercetin glucuronide from PhytoLab (Vestenbergsgreuth, Germany). The other solvents and reagents, if not otherwise specified, were acquired from P.O.Ch. (Gliwice, Poland).
Preparation of crude extract of sunflower seeds

The sunflower kernels were ground in a coffee mill (BSH Bosch \& Siemens, Munich, Germany) and defatted with hexane using a Soxhlet apparatus. A $25 \mathrm{-g}$ portion of defatted flour was weighed into dark glass bottles and suspended in $200 \mathrm{~mL}$ of $80 \%(\mathrm{v} / \mathrm{v})$ aqueous methanol and shaken at $60{ }^{\circ} \mathrm{C}$ for $15 \mathrm{~min}$ in a water bath (Elpan, Lubawa, Poland). The cooled supernatant was filtered, and the residue was extracted twice more. Methanol from the combined filtrates was evaporated under vacuum at $40{ }^{\circ} \mathrm{C}$ (Rotavapor R-200, Büchi Labortechnik, Flawil, Switzerland), whereas water residue was lyophilized for $\sim 48 \mathrm{~h}$ at $-70{ }^{\circ} \mathrm{C}$ and 0.013 mbar (Lyph Lock 6 freeze dry system, Labconco, Kansas City, USA). The extraction was carried out in triplicate.

Fractionation of crude extract

A suspension of the extract $(1 \mathrm{~g}$ in $8 \mathrm{~mL}$ of methanol) was loaded onto a column filled with Sephadex LH-20 gel $(2 \times 60 \mathrm{~cm})$. The chromatographic separation was performed using methanol as a mobile phase, which was delivered to the column by a LKB 2132 MicroPerpex peristaltic pump (Pharmacia LKB, Uppsala, Sweden) at a flow rate of $0.7 \mathrm{~mL} / \mathrm{min}$. The void volume of the eluate $V_{0}=240 \mathrm{~mL}$ was discarded, and then $10 \mathrm{~mL}$ of fractions was collected using a RediFrac fraction collector (Pharmacia LKB). The individual fractions were analyzed by absorbance measurement at $\lambda=320 \mathrm{~nm}$ and after reaction with Folin-Ciocalteu's reagent (FCR) - the absorbance of the products formed was recorded at $\lambda=725 \mathrm{~nm}$ using a spectrophotometer DU-7500 (Beckman Instruments, Fullerton, USA). Subsequently, based on the prepared chromatograms of separation, the eluates were combined into six main fractions, and methanol was evaporated using a rotary evaporator.

\section{Determination of total phenolic content (TPC)}

The TPC of the main fractions was determined using reaction with FCR [14]. Briefly, to $0.25 \mathrm{~mL}$ of methanolic solutions of fractions, $0.25 \mathrm{~mL}$ of FCR (diluted twofold), $0.5 \mathrm{~mL}$ of saturated solution of sodium carbonate and $4 \mathrm{~mL}$ of water were added. After a 25-min quiescent period, the reaction mixture was centrifuged (MPW-210, MPW Med. Instruments, Warsaw, Poland) for $5 \mathrm{~min}$ at $5,000 \times g$, and the absorbance of supernatants at $\lambda=725 \mathrm{~nm}$ was read using a DU-7500 spectrophotometer. $(+)$-Catechin and 5-O-caffeoylquinic acid were used to prepare the standard curves. The results were expressed as milligram of standard equivalents (eq) per gram of fraction. 
Identification and quantification of phenolic compounds

The samples were dissolved in 80:20 (v/v) methanol/water, filtered through a $0.45-\mu \mathrm{m}$ cellulose acetate filter (Millipore, Billerica, USA) and analyzed using a Waters HPLC system (Milford, USA) consisting of an autoinjector, a quaternary pump, a 2001 photodiode array detector (PDA) and Millenium 32 chromatography manager software. The samples were injected into a Nova-Pak $\mathrm{C}_{18}$ column $(3.9 \times 300 \mathrm{~mm}$; particle size, $4 \mu \mathrm{m}$; Waters $)$ and eluted for $110 \mathrm{~min}$ in a gradient system consisting of solvent $\mathrm{A}$, water/acetic acid $(98: 2 \mathrm{v} / \mathrm{v})$, and solvent $\mathrm{B}$, water/acetonitrile/acetic acid (78:20:2 v/v/v). The gradient profile was the following: 0-55 $\mathrm{min}, 100-20 \% \mathrm{~A}$; 55-70 $\mathrm{min}$, 20-10\% A; 70-80 min, 10-5\% A; and 80-110 min, $100 \% \mathrm{~B}$. The flow rate was $1 \mathrm{~mL} / \mathrm{min}$ and injection volume $30 \mu \mathrm{L}$. The column was re-equilibrated between sample injections with $10 \mathrm{~mL}$ of acetonitrile and $25 \mathrm{~mL}$ of mobile phase A. The diode array detection was performed by scanning over a wavelength range from 210 to $400 \mathrm{~nm}$ at an acquisition of $1 \mathrm{~s}$. The quantification of individual compounds was carried out using a calibration curve of the corresponding standard or, when reference compound was not available, of structurally related substances. Samples were analyzed in duplicate.

The mass spectra were obtained using a Hewlett Packard Model 1100 Series LC/MSD system (Palo Alto, USA) equipped with an electrospray ionization (ESI) interface. The solvent gradient and column employed were identical to those for HPLC-PAD analyses. The ESI conditions were as follows: $\mathrm{N}_{2}$ was used both as the nebulizing gas at $275 \mathrm{kPa}$ and as drying gas (flow rate, $10 \mathrm{~L} / \mathrm{min}$; temperature, $340{ }^{\circ} \mathrm{C}$ ); voltage at the capillary entrance, $4,000 \mathrm{~V}$; and variable fragmentation voltage, $100 \mathrm{~V}(\mathrm{~m} / \mathrm{z}$ 200-1,000) and $250 \mathrm{~V}(\mathrm{~m} / \mathrm{z}, 1,000-2,500)$. Negative-ion mass spectra of the column eluate were recorded from $\mathrm{m} / \mathrm{z}$ of 100 to $\mathrm{m} / \mathrm{z}$ of 2,500 .

\section{Radical-scavenging activity}

DPPH-scavenging activity of individual fractions was measured according to the method described by Yen and Chen [15]. From methanolic solutions of fractions at a concentration of $1 \mathrm{mg} / \mathrm{mL}, 0.1 \mathrm{~mL}$ was pipetted into the tube containing $0.25 \mathrm{~mL}$ of $1 \mathrm{mM}$ methanolic solution of DPPH and $2 \mathrm{~mL}$ of methanol. The mixture was vortexed immediately (Vortex Genie2, Scientific Industries INC., Bohemia, USA) and allowed to stand at $20{ }^{\circ} \mathrm{C}$ in the darkness for $20 \mathrm{~min}$. Then, the absorbance at $\lambda=517 \mathrm{~nm}$ was recorded. The ability to scavenge the DPPH radical was calculated using the following equation: scavenging effect $(\%)=\left[\left(A_{0}-A_{1}\right) / A_{0}\right] \times 100$, where $A_{0}$ is the absorbance of the DPPH solution and $A_{1}$ is the absorbance in the presence of the sample. Scavenging effect versus concentration of fractions (mg/assay) was plotted. The $\mathrm{EC}_{50}$ value, defined as the amount of antioxidant $(\mathrm{mg})$ necessary to inhibit the initial DPPH by $50 \%$, was read from the plot.

Evaluation of Trolox equivalent antioxidant capacity (TEAC)

The TEAC of fractions was determined using the ABTS radical cation decolorization assay [16]. ABTS $^{+}$was prepared by reaction of $192 \mathrm{mg}$ ABTS and $33 \mathrm{mg}$ potassium persulfate in $50 \mathrm{~mL}$ water. The mixture was stirred overnight in the darkness at room temperature; afterward, the solution was diluted with methanol up to a final absorbance of $0.70 \pm 0.02$ at $734 \mathrm{~nm}$. Two milliliters portions of $\mathrm{ABTS}^{+}$was vortexed with $20 \mu \mathrm{L}$ of methanolic solution of fractions. Samples were incubated at $30{ }^{\circ} \mathrm{C}$ using a block heater (TH-24, Meditherm, Warsaw, Poland), and the absorbance was recorded at $\lambda=734 \mathrm{~nm}$ after 6 min, strictly. Trolox was used to obtain the standard curve. The results were expressed in $\mathrm{mmol}$ of Trolox eq/g of fraction.

\section{Reducing power assay}

The reducing power was determined according to the method of Oyaizu [17]. The fraction solutions $(1.0 \mathrm{~mL}$, $0.2-1.0 \mathrm{mg} / \mathrm{mL}$ ) were mixed with $2.5 \mathrm{~mL}$ of phosphate buffer $(0.2 \mathrm{M}, \mathrm{pH} 6.6)$ and $2.5 \mathrm{~mL}$ of potassium ferricyanide $(1 \%)$. Then, the mixture was incubated at $50{ }^{\circ} \mathrm{C}$ for $20 \mathrm{~min}$ (TH-24, Meditherm). Following this, a portion of trichloroacetic acid $(2.5 \mathrm{~mL}, 10 \%)$ was added. A $2.5 \mathrm{~mL}$ of the aliquot was mixed with $2.5 \mathrm{~mL}$ of distilled water and $0.5 \mathrm{~mL}$ of $0.1 \%$ ferric chloride. The absorbance of the mixture was recorded at $\lambda=700 \mathrm{~nm}$. The absorbance versus concentration of fractions (mg/assay) was plotted.

Statistical analysis

The mean values and standard deviations $(n=3)$ for all conducted analysis were calculated using Microsoft Excel 2000 software (Microsoft Office Excel for Windows, Microsoft Co., Redmond, USA). The linear correlation coefficients between the total phenolic content and antioxidant activity were calculated by Pearson test using GraphPad Prism version 5.02 for Windows (GraphPad Software, San Diego, USA). TPC and TEAC values were analyzed by a one-way ANOVA statistical model with Tukey's post hoc test. Differences were considered significant at $P<0.05$. 


\section{Results and discussion}

The phenolic compounds from sunflower seed crude extracts were fractionated into six fractions (I-VI) (Fig. 1). The yield of separated fractions in the extract and the total phenolic content of fractions are given in Table 1. The maximum optical density determined after reaction with FCR for fraction I was higher than for the other fractions. In turn, when detection was carried out at $320 \mathrm{~nm}$, the highest optical density value was noted for fraction V. Fraction I accounted for $58.4 \%$ of extract; however, it was characterized by a very low content of phenolic compounds amounting to $18.2 \mathrm{mg}(+)$-catechin eq/g only. Since crude extract of sunflower seeds was obtained using aqueous methanol, besides phenolic compounds, also soluble sugars might have been extracted. The carbohydrates are eluted from Sephadex LH-20 column chromatography with methanol at the beginning of separation [18]. Amarowicz et al. [19, 20] and Arfan et al. [21] noted low content of

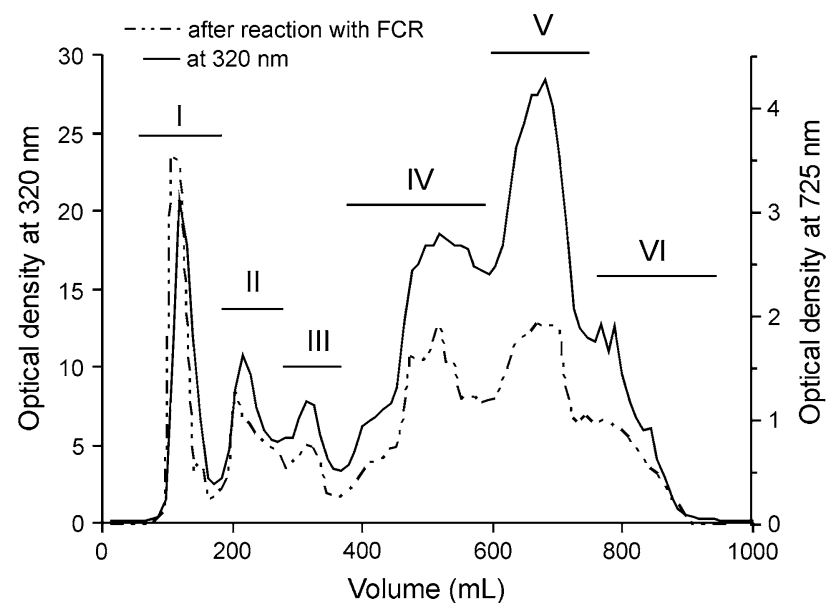

Fig. 1 Separation of sunflower seed crude extract using Sephadex LH-20 column chromatography; FCR Folin-Ciocalteu's reagent

Table 1 Yield of sunflower seed fractions and total phenolic content of individual fractions

\begin{tabular}{lccc}
\hline $\begin{array}{l}\text { Fraction } \\
\text { number }\end{array}$ & $\begin{array}{l}\text { Yield } \\
(\%)\end{array}$ & \multicolumn{2}{l}{ Total phenolic content } \\
\cline { 3 - 4 } & & $\begin{array}{l}\text { (mg }(+)- \\
\text { catechin eq/g) }\end{array}$ & $\begin{array}{l}(\mathrm{mg} \mathrm{5-O} \text {-caffeoylquinic } \\
\text { acid eq/g) }\end{array}$ \\
\hline I & 58.4 & $18.2 \pm 0.62^{\mathrm{a}}$ & $24.5 \pm 0.90^{\mathrm{a}}$ \\
II & 12.4 & $108 \pm 0.91^{\mathrm{b}}$ & $150 \pm 1.32^{\mathrm{b}}$ \\
III & 2.7 & $305 \pm 2.47^{\mathrm{c}}$ & $407 \pm 3.46^{\mathrm{c}}$ \\
IV & 8.5 & $470 \pm 0.96^{\mathrm{d}}$ & $645 \pm 1.38^{\mathrm{d}}$ \\
V & 8.9 & $485 \pm 5.39^{\mathrm{e}}$ & $666 \pm 7.28^{\mathrm{e}}$ \\
VI & 4.2 & $378 \pm 5.71^{\mathrm{f}}$ & $511 \pm 8.25^{\mathrm{f}}$ \\
\hline
\end{tabular}

Results are mean values \pm standard deviations $(n=3)$

Means with different superscript letters (a-f), within a column, are significantly different at $P<0.05$ phenolic compounds in the first fractions obtained from chromatographic separation of crude extracts of legume seeds and Litsea monopetala bark on Sephadex LH-20.

Fractions II-VI were characterized by high total phenolic content (Table 1). The highest content was noted for fractions IV and V, 470 and $485 \mathrm{mg}(+)$-catechin eq/g, respectively. The yields of those fractions were also appreciable. They accounted for 8.5 and $8.9 \%$ of the total weight of extract, respectively. Generally, the total phenolic content of fractions II-VI was several times higher in comparison with fractions obtained from canola [18], everlasting pea, faba bean, broad bean [22], pea [19] and lentil seeds [20].

Since phenolic compounds of sunflower seeds comprise mainly 5-O-caffeoylquinic acid [8-10], the results of total phenolic content were additionally expressed as 5-O-caffeoylquinic acid eq (Table 1). The results obtained in that manner were approximately 1.4 times higher in comparison with those expressed as (+)-catechin eq. A significant correlation (0.978; $P=0.0007)$, as determined by the Pearson test, was found between the content of phenolic compounds determined by FCR assay and sum of individual phenolic compounds quantified by HPLC-MS(ESI) (Table 2).

In Table 3, the $\lambda_{\max }$ of UV spectra and characteristic ions of phenolic compounds identified in the sunflower seed fractions are presented. The trans-ferulic acid, 5-Ocaffeoylquinic acid, quercetin rutinoside and quercetin glucuronide (compounds 1, 5, 29, 30) were identified by comparison of their retention times and UV spectra with respective reference substances. The cis-ferulic acid (2) was identified in agreement with data from Dueñas et al. [23].

The presence of 5-O-caffeoylquinic acid (5) was confirmed by HPLC-MS(ESI) analysis. This compound, present in all analyzed fractions, showed a deprotonated molecular ion $[\mathrm{M}-\mathrm{H}]^{-}$at a $\mathrm{m} / \mathrm{z}$ of 353 , main fragment ion at a $m / z$ of 191 (quinic acid moiety) and a fragment ion at a $\mathrm{m} / \mathrm{z}$ of 179 (caffeic acid moiety) with low intensity, which is in accordance with literature data [24, 25]. Compounds 3 (fraction VI) and 4 (fractions III-VI) produced similar parent ion at a $\mathrm{m} / \mathrm{z}$ of 353 corresponding to the molecular formula $\mathrm{C}_{16} \mathrm{H}_{17} \mathrm{O}_{9}$. Since main fragment ions of those compounds were at a $\mathrm{m} / \mathrm{z}$ of 191 and a $\mathrm{m} / \mathrm{z}$ of 179 (compounds 3) and a $\mathrm{m} / \mathrm{z}$ of 173 (compounds 4), they were assigned to 3-O-caffeoylquinic acid and 4- $O$-caffeoylquinic acid, respectively, taking into account the different intensity of the fragment ions $[24,25]$. The parent ion at a $\mathrm{m} / \mathrm{z}$ of 337 was characteristic of compounds $6,7,8$. They were identified as 3-O-p-coumaroylquinic acid (fractions II-V), 4-O- - -coumaroylquinic acid (fraction III) and 5-O$p$-coumaroylquinic acid (fraction III), due to the presence of the main fragment ions $[p \text {-coumaric acid }-\mathrm{H}]^{-}$at a $\mathrm{m} / \mathrm{z}$ 
Table 2 Pearson's correlation coefficients between total phenolic content (TPC), sum of phenolic compounds determined by HPLC-MS(ESI) and data obtained in antioxidant activity tests of sunflower seed fractions

\begin{tabular}{lllll}
\hline & TPC & TEAC $^{\mathrm{a}}$ & Reducing power $^{\mathrm{b}}$ & EC $_{50}^{\mathrm{c}}$ \\
\hline Sum of phenolics & $0.978(P=0.0007)$ & $0.992(P=0.0001)$ & $0.968(P=0.002)$ & $-0.868(P=0.025)$ \\
TPC & & $0.971(P=0.0012)$ & $0.988(P=0.0002)$ & $-0.907(P=0.013)$ \\
TEAC $^{\mathrm{a}}$ & & & $0.978(P=0.001)$ & $-0.896(P=0.016)$ \\
Reducing power $^{\mathrm{b}}$ & & & $-0.908(P=0.012)$ \\
\hline
\end{tabular}

a TEAC, Trolox equivalent antioxidant capacity determined by ABTS assay

${ }^{\mathrm{b}}$ Reducing power at a concentration $1 \mathrm{mg}$ of fraction per assay

${ }^{c} \mathrm{EC}_{50}$ values for DPPH radicals inhibition

Table $3 \lambda_{\max }$ of UV spectra and characteristic ions of phenolic compounds detected in fractions of sunflower seeds

\begin{tabular}{|c|c|c|c|c|c|c|c|c|c|c|}
\hline \multirow[t]{2}{*}{ No. compound } & \multirow[t]{2}{*}{ Compounds } & \multirow[t]{2}{*}{$\lambda_{\max }(\mathrm{nm})$} & \multirow[t]{2}{*}[\mathrm{M}-\mathrm{H}]{$^{-}(m / z)$} & \multirow[t]{2}{*}{ Fragment ions $(\mathrm{m} / \mathrm{z})$} & \multicolumn{6}{|c|}{ Fraction } \\
\hline & & & & & $\mathrm{I}$ & II & III & IV & $\mathrm{V}$ & VI \\
\hline 1 & trans-Ferulic acid & 328 & 193 & - & + & + & + & - & - & - \\
\hline 2 & cis-Ferulic acid & 312 & 193 & - & - & - & - & + & + & + \\
\hline 3 & 3-O-Caffeoylquinic acid & 326 & 353 & $191,179,135$ & - & - & - & - & - & + \\
\hline 4 & 4-O-Caffeoylquinic acid & 326 & 353 & $191,179,173,135$ & - & - & + & + & + & + \\
\hline 5 & 5-O-Caffeoylquinic acid & 326 & 353 & $191,179,135$ & + & + & + & + & + & + \\
\hline 6 & 3-O-p-Coumaroylquinic acid & 316 & 337 & $191,163,119$ & - & + & + & + & + & - \\
\hline 7 & 4-O-p-Coumaroylquinic acid & 316 & 337 & $191,173,163$ & - & - & + & - & - & - \\
\hline 8 & 5-O-p-Coumaroylquinic acid & 316 & 337 & 191,163 & - & - & + & - & - & - \\
\hline 9 & Dicaffeoylquinic acid (1) & 326 & 515 & $353,179,173$ & - & + & - & - & - & - \\
\hline 10 & Dicaffeoylquinic acid (2) & 326 & 515 & $353,191,179,135$ & - & + & - & - & + & - \\
\hline 11 & Dicaffeoylquinic acid (3) & 326 & 515 & $353,179,135$ & - & + & - & - & - & - \\
\hline 12 & Dicaffeoylquinic acid (4) & 326 & 515 & 353,335 & - & - & - & - & - & + \\
\hline 13 & Dicaffeoylquinic acid (5) & 326 & 515 & 353,335 & - & - & - & + & - & + \\
\hline 14 & Dicaffeoylquinic acid (6) & 326 & 515 & $353,191,173$ & - & - & - & - & - & + \\
\hline 15 & Caffeoyl-dimethoxycinnamoylquinic acid (1) & 328 & 543 & $381,335,133$ & - & + & + & + & - & - \\
\hline 16 & Caffeoyl-dimethoxycinnamoylquinic acid (2) & 328 & 543 & $381,335,133$ & - & + & + & + & - & - \\
\hline 17 & Caffeoyl-dimethoxycinnamoylquinic acid (3) & 326 & 543 & 381,335 & - & - & - & + & + & - \\
\hline 18 & Caffeoyl-dimethoxycinnamoylquinic acid (4) & 326 & 543 & 381,335 & - & - & - & - & + & + \\
\hline 19 & Caffeoyl-dimethoxycinnamoylquinic acid (5) & 326 & 543 & $335,179,173$ & - & - & - & + & + & - \\
\hline 20 & Caffeoylferuloylquinic acid & 325 & 529 & 193 & - & + & - & - & - & - \\
\hline 21 & Ferulic acid dehydrotrimer (1) & 324 & 563 & 193 & - & - & + & - & - & - \\
\hline 22 & Ferulic acid dehydrotrimer (2) & 326 & 563 & 193 & + & + & - & - & - & - \\
\hline 23 & Ferulic acid dehydrotrimer (3) & 326 & 563 & 193 & - & - & + & - & - & - \\
\hline 24 & Ferulic acid dehydrotrimer (4) & 326 & 563 & 193 & - & + & - & - & - & - \\
\hline 25 & Ferulic acid dehydrotrimer (5) & 329 & 563 & 193 & - & + & - & - & + & + \\
\hline 26 & Ferulic acid dehydrotrimer (6) & 326 & 563 & 193 & - & - & - & + & + & + \\
\hline 27 & Quercetin derivative & 257,353 & - & 301 & + & - & - & - & - & - \\
\hline 28 & Quercetin diglycoside & 257,355 & 595 & 301 & - & - & + & - & - & - \\
\hline 29 & Quercetin rutinoside & 256,348 & 609 & 301 & - & - & - & + & - & - \\
\hline 30 & Quercetin glucuronide & 258,351 & 477 & 301 & - & - & - & - & + & + \\
\hline 31 & Flavanone & 290,332 & 579 & 449 & - & - & - & + & - & - \\
\hline
\end{tabular}

of 163 , [quinic acid- $\left.-\mathrm{H}_{2} \mathrm{O}-\mathrm{H}\right]^{-}$at a $\mathrm{m} / \mathrm{z}$ of 173 and [quinic acid- $-\mathrm{H}]^{-}$at a $\mathrm{m} / \mathrm{z}$ of 191 , respectively [24]. The presence of four monoacyl quinic acids (out of six isomers detected in the present study) was noted in sunflower seeds in previous analyses: three isomers of caffeoylquinic acid and 5-O-p-coumaroylquinic acid $[8,10,12]$. However, to our 
knowledge, 3-O-p-coumaroylquinic acid and 4-O-p-coumaroylquinic acid were identified as sunflower seed phenolic compounds for the first time.

Six compounds 9, 10,11,12, 13 and 14 exhibiting a [M$\mathrm{H}]^{-}$ion at a $\mathrm{m} / \mathrm{z}$ of 515 and fragment ions such as [M-

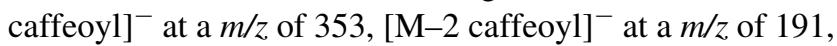
[caffeic acid- $\mathrm{H}]^{-}$at a $\mathrm{m} / z$ of 179 , [quinic acid- $\mathrm{H}_{2} \mathrm{O}-$ $\mathrm{H}^{-}$at a $\mathrm{m} / \mathrm{z}$ of 173 and [caffeic acid- $\left.-\mathrm{CO}_{2} \mathrm{H}\right]^{-}$at a $\mathrm{m} / \mathrm{z}$ of 135 were assigned to dicaffeoylquinic acid isomers [25]. Aramendia et al. [12] and Weisz et al. [10] reported the presence of three dicaffeoylquinic acid isomers among phenolic compounds of sunflower seeds. They were identified as 3,4-di- $O$-caffeoylquinic, 3,5-di- $O$-caffeoylquinic and 4,5-di$O$-caffeoylquinic acids. In turn, Pedrosa et al. [8] noted the presence of four dicaffeoylquinic acid isomers in sunflower seeds: three aforementioned, and the other was presumably 1,5-di- $O$-caffeoylquinic acid. The fragment ion at a $\mathrm{m} / z$ of 335 in the compounds 12 and 13 suggested the presence of the isomers 1,3-di- $O$-caffeoylquinic and 1,4-di- $O$-caffeoylquinic, reported by Clifford et al. [25].

The compound 20 present in fraction II was identified as one of the caffeoylferuloylquinic acid isomers on the basis of $[\mathrm{M}-\mathrm{H}]^{-}$ion at a $\mathrm{m} / \mathrm{z}$ of 529 and fragment ion at a $\mathrm{m} / \mathrm{z}$ of 193 (ferulic acid moiety) [24]. Among diacyl quinic acids, the presence of five caffeoyl-dimethoxycinnamoylquinic acid isomers was noted in sunflower seeds. Isomers 15 and 16 were detected in fractions II-IV, and isomers 17, 18 and 19 in fractions IV-VI. The identification was performed on the basis of the occurrence of deprotonated molecular ion $[\mathrm{M}-\mathrm{H}]^{-}$at a $\mathrm{m} / \mathrm{z}$ of 543 and fragmentation of the parent ion, which was in accordance with literature data [26]. Heretofore, caffeoyl-dimethoxycinnamoylquinic acid isomers were not recognized as phenolic compounds of sunflower seeds.

Compounds 21, 22, 23, 24, 25 and 26 were tentatively identified as related to ferulic acid dehydrotrimers. These compounds displayed a UV spectrum characteristic of ferulic acid-related structures, a fragment ion at a $\mathrm{m} / \mathrm{z}$ of 193 (ferulic acid moiety) and an ion $[\mathrm{M}-\mathrm{H}]^{-}$at a $\mathrm{m} / \mathrm{z}$ of 563 which could correspond to different dehydrotriferulic acids [27]. In previous study on sunflower seeds phenolic compounds, Pedrosa et al. [8] noted the presence of compound showing a parent ion at a $\mathrm{m} / \mathrm{z}$ of 563 with fragmentation yielded ions at $\mathrm{m} / \mathrm{z}$ of 353,191 and 179 , but the authors did not identify the structure of that compound.

Among non-esterified phenolic compounds, only trans and cis isomers of ferulic acid (compounds 1 and 2, respectively) were identified in the fractions: trans-ferulic acid in fractions I-III, and cis-ferulic acid in IV-VI. Weisz et al. [10] detected two non-esterified phenolic acids such as caffeic and ferulic using HPLC-DAD/ESI-MS ${ }^{\mathrm{n}}$ analysis, whereas Pedrosa et al. [8] additionally found trace amounts of $p$-coumaric, $p$-hydroxybenzoic, protocatechuic and syringic acids by RP-HPLC analysis.

In the fractions from sunflower seeds, a few compounds belonging to flavonoids were also identified. Compounds 27 and 28 due to the characteristic $\lambda_{\max }$ of UV spectra and fragment ion at a $\mathrm{m} / \mathrm{z}$ of 301 (quercetin moiety) were classified as quercetin derivatives, with compound 28 being probably a diglycoside, but no further identifications were possible. Fraction IV contained the quercetin rutinoside (29) $(\mathrm{m} / z, 609,301)$, and in fractions V and VI, one of quercetin glucuronide isomers (30) was identified due to the occurrence of deprotonated molecular ion $[\mathrm{M}-\mathrm{H}]^{-}$at a $\mathrm{m} / \mathrm{z}$ of 477 and fragment ion at a $\mathrm{m} / \mathrm{z}$ of 301 (quercetin moiety) [28]. Furthermore, in fraction IV, a flavanone (compound 31) with $\lambda_{\max }$ of UV spectrum at 290 and $332 \mathrm{~nm}$ was tentatively identified.

The contents of individual phenolic compounds identified in fractions of sunflower seeds are presented in Table 4 . The 5-O-caffeoylquinic acid was the predominant compound in fractions I, II, III, IV and V. It accounted for 89.0, 94.9, 85.1, 93.4 and $95.8 \%$ of the sum of phenolic compounds identified in individual fraction, respectively. However, the content of 5-O-caffeoylquinic acid in those fractions varied from 6.98 to $308.9 \mathrm{mg} / \mathrm{g}$. It was calculated that 5-O-caffeoylquinic acid present in fraction IV and $\mathrm{V}$ amounted to $47.6 \mathrm{mg} / \mathrm{g}$ of extract, which accounts for $76.1 \%$ of total amount of that compound eluted from sunflower seeds extract loaded on Sephadex LH-20 column. The other fractions contained from 1.4 to $5.2 \mathrm{mg}$ of $5-O-$ caffeoylquinic acid per gram of extract. The estimated content of 5-O-caffeoylquinic acid is in accordance with the literature. Weisz et al. [10] noted that this compound might comprise from 66 to $73 \%$ of phenolic compounds extracted from defatted kernels of non-oilseeds and oilseeds of sunflower. Pedrosa et al. [8] obtained more diversified results ranging within $43-71 \%$ for kernels of different genotypes. In the present study, 5-O-caffeoylquinic acid was not the only predominant compound in fraction VI. That constituent accounted for $18.2 \%$ of the sum of phenolic compounds of that fraction. Diacyl quinic acids dominated in fraction VI. They comprised $76.6 \%$ of the sum of phenolic compounds of fraction VI. Among them, one of the dicaffeoylquinic acid isomers $(79.74 \mathrm{mg} / \mathrm{g}$ of fraction) and one of the caffeoyl-dimetoxycinnamoylquinic acids $(61.69 \mathrm{mg} / \mathrm{g}$ of fraction) were predominant. Weisz et al. [10] showed that the ratio of monocaffeoylquinic acids content to dicaffeoylquinic acids content for sunflower kernels ranged from 5.6:1 to 16.9:1. The ratio of those compounds in our study was 17.5:1. The majority $(75.8 \%)$ of ferulic acid dehydrotrimer isomers was noted in fraction III. This fraction contained also the highest amount of flavonoids and p-coumaroylquinic acid isomers. 
Table 4 Content of identified phenolic compounds in fractions of sunflower seeds (mg/g of fraction)

\begin{tabular}{|c|c|c|c|c|c|c|c|}
\hline \multirow[t]{2}{*}{ Class of phenolics } & \multirow[t]{2}{*}{ Compounds } & \multicolumn{6}{|c|}{ Fraction } \\
\hline & & I & II & III & IV & $\mathrm{V}$ & VI \\
\hline \multirow[t]{3}{*}{ Non-esterified phenolic acids } & trans-Ferulic acid & 0.02 & 0.02 & 1.65 & - & - & - \\
\hline & cis-Ferulic acid & - & - & - & 0.67 & 0.95 & 2.30 \\
\hline & Sum & 0.02 & 0.02 & 1.65 & 0.67 & 0.95 & 2.30 \\
\hline \multirow[t]{7}{*}{ Monoacyl quinic acids } & 3-O-Caffeoylquinic acid & - & - & - & - & - & 1.63 \\
\hline & 4- $O$-Caffeoylquinic acid & - & - & 1.42 & 0.23 & 0.39 & 1.79 \\
\hline & 5-O-Caffeoylquinic acid & 6.98 & 42.1 & 156.9 & 236.3 & 308.9 & 34.11 \\
\hline & 3-O-p-Coumaroylquinic acid & - & 0.46 & 6.04 & 2.58 & 0.99 & - \\
\hline & 4-O-p-Coumaroylquinic acid & - & - & 1.18 & - & - & - \\
\hline & 5-O-p-Coumaroylquinic acid & - & - & 1.01 & - & - & - \\
\hline & Sum & 6.98 & 42.56 & 166.6 & 239.1 & 310.3 & $\mathbf{3 7 . 5 3}$ \\
\hline \multirow[t]{13}{*}{ Diacyl quinic acids } & Dicaffeoylquinic acid (1) & - & 0.23 & - & - & - & - \\
\hline & Dicaffeoylquinic acid (2) & - & 0.11 & - & - & 0.45 & - \\
\hline & Dicaffeoylquinic acid (3) & - & 0.25 & - & - & - & - \\
\hline & Dicaffeoylquinic acid (4) & - & - & - & 0.34 & - & 1.67 \\
\hline & Dicaffeoylquinic acid (5) & - & - & - & - & - & 0.58 \\
\hline & Dicaffeoylquinic acid (6) & - & - & - & - & - & 79.74 \\
\hline & Caffeoyl-dimethoxycinnamoylquinic acid (1) & - & 0.07 & 0.89 & 2.83 & - & - \\
\hline & Caffeoyl-dimethoxycinnamoylquinic acid (2) & - & 0.24 & 1.21 & 0.43 & - & - \\
\hline & Caffeoyl-dimethoxycinnamoylquinic acid (3) & - & - & - & 2.81 & 4.31 & - \\
\hline & Caffeoyl-dimethoxycinnamoylquinic acid (4) & - & - & - & 2.54 & 3.24 & 61.69 \\
\hline & Caffeoyl-dimethoxycinnamoylquinic acid (5) & - & - & - & - & 0.26 & - \\
\hline & Caffeoylferuloylquinic acid & - & 0.01 & - & - & - & - \\
\hline & Sum & $\mathbf{0 . 0 0}$ & 0.91 & 2.1 & 8.95 & 8.26 & 143.68 \\
\hline \multirow[t]{7}{*}{ Ferulic acid dehydrotrimers } & Ferulic acid dehydrotrimer (1) & 0.22 & - & 1.85 & - & - & - \\
\hline & Ferulic acid dehydrotrimer (2) & - & 0.65 & 2.47 & - & - & - \\
\hline & Ferulic acid dehydrotrimer (3) & - & 0.11 & - & - & - & - \\
\hline & Ferulic acid dehydrotrimer (4) & - & 0.13 & - & - & - & - \\
\hline & Ferulic acid dehydrotrimer (5) & 0.01 & - & - & - & 0.04 & - \\
\hline & Ferulic acid dehydrotrimer (6) & - & - & - & 0.1 & 0.06 & 0.06 \\
\hline & Sum & 0.23 & $\mathbf{0 . 8 9}$ & 4.32 & 0.1 & 0.1 & 0.06 \\
\hline \multirow[t]{6}{*}{ Flavonoids } & Quercetin derivative & 0.61 & - & - & - & - & - \\
\hline & Quercetin diglycoside & - & - & 9.84 & - & - & - \\
\hline & Quercetin rutinoside & - & - & - & 2.12 & - & - \\
\hline & Quercetin glucuronide & - & - & - & - & 2.72 & 4.08 \\
\hline & Flavanone & - & - & - & 1.94 & - & - \\
\hline & Sum & 0.61 & 0.00 & 9.84 & 4.06 & 2.72 & 4.08 \\
\hline
\end{tabular}

DPPH-scavenging activity of sunflower seed fractions is shown in Fig. 2. The fractions III-VI at the highest concentration $(0.1 \mathrm{mg} /$ assay) inactivated more than $90 \%$ of DPPH radicals. In turn, fractions I and II were much weaker scavengers of DPPH. The amount of the fraction necessary to inhibit the initial DPPH by $50 \%\left(\mathrm{EC}_{50}\right.$ value) was the lowest for fraction VI and amounted to 0.024 (Table 5). Fraction $\mathrm{V}$ was characterized by similar $\mathrm{EC}_{50}$ value, whereas fractions III and IV by higher values. In the assay conditions, fractions I and II inhibited DPPH radicals by less than $50 \%$. The ABTS cation radical-reducing ability of sunflower seed fractions was expressed as TEAC values and compiled in the Table 5. The TEAC values varied from $0.10 \mathrm{mmol}$ Trolox $\mathrm{eq} / \mathrm{g}$ for fraction I to $2.21 \mathrm{mmol}$ Trolox eq/g for fraction $\mathrm{V}$, and the $\mathrm{ABTS}^{+{ }_{-}}$ reducing capacity was increasing in the following order: $\mathrm{I}<\mathrm{II}<\mathrm{III} \leq \mathrm{VI} \leq \mathrm{IV}<\mathrm{V} \quad(P<0.05) . \quad$ The highest reducing power, similarly as the capacity to scavenge ABTS $^{+}$, was noted for fraction V (Fig. 3). Fractions IV and VI showed comparable ability to reduce $\mathrm{Fe}^{3+}$ / 


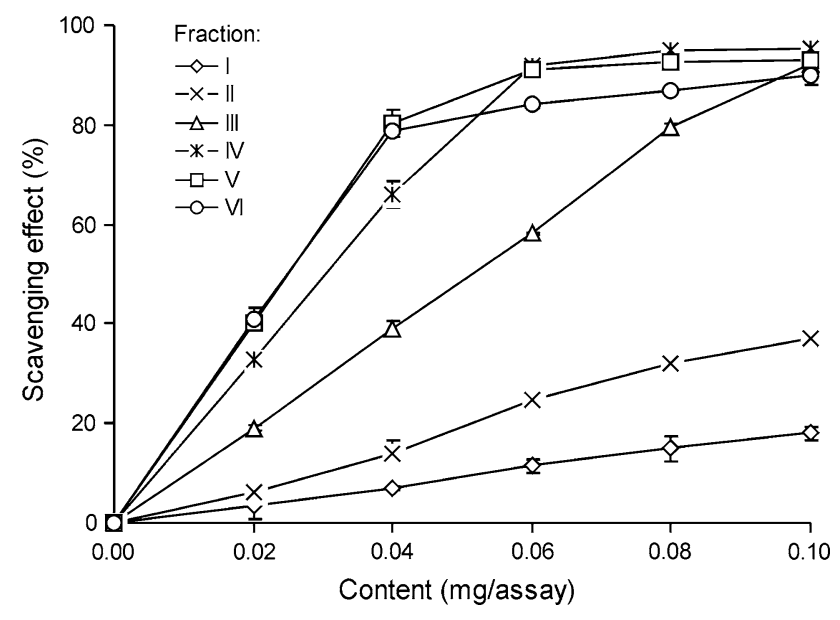

Fig. 2 Scavenging effect of sunflower seed fractions on 1,1-diphenyl2-picrylhydrazyl radical (DPPH)

Table $5 \mathrm{ABTS}^{-+}$scavenging activity expressed as Trolox equivalent antioxidant capacity (TEAC) and $\mathrm{DPPH}$-scavenging activity expressed as $\mathrm{EC}_{50}$ values of sunflower seed fractions

\begin{tabular}{llc}
\hline Fraction number & TEAC $(\mathrm{mmol}$ Trolox eq/g) & EC $_{50}(\mathrm{mg})$ \\
\hline I & $0.10 \pm 0.012^{\mathrm{a}}$ & $>0.1$ \\
II & $0.51 \pm 0.019^{\mathrm{b}}$ & $>0.1$ \\
III & $1.32 \pm 0.176^{\mathrm{c}}$ & 0.051 \\
IV & $1.59 \pm 0.159^{\mathrm{d}}$ & 0.030 \\
V & $2.21 \pm 0.217^{\mathrm{e}}$ & 0.025 \\
VI & $1.47 \pm 0.046^{\mathrm{c}, \mathrm{d}}$ & 0.024 \\
\hline
\end{tabular}

Results are mean values \pm standard deviations $(n=3)$

Means with different superscript letters (a-e), within a column, are significantly different at $P<0.05$

ferricyanide complex to the ferrous form in the whole range of concentration/assay. The reducing power determined for fractions I, II and III was lower in comparison with fractions $\mathrm{V}$ by 11,4 and 1.5 times, respectively. The results of all applied methods of antioxidant activity testing were significantly correlated with the content of phenolic compounds in fractions (Table 2). High Pearson's correlation coefficients were noted also between TEAC, reducing power and $\mathrm{EC}_{50}$ values (Table 2).

Since 5-O-caffeoylquinic acid is a major phenolic constituent of fractions I-V (Table 4), their antioxidant activity (Table 5; Figs. 2, 3) can be attributed to this compound. It is well known that $5-O$-caffeoylquinic acid is able to scavenge free radicals and inhibit oxidation of various lipid substrates [29]. Its antioxidant activity is comparable to or only slightly lower (depending on the method used) than the activity of caffeic acid and significantly higher than other hydroxycinnamic acids [29-32]. It is determined by the structure of 5-O-caffeoylquinic acid,

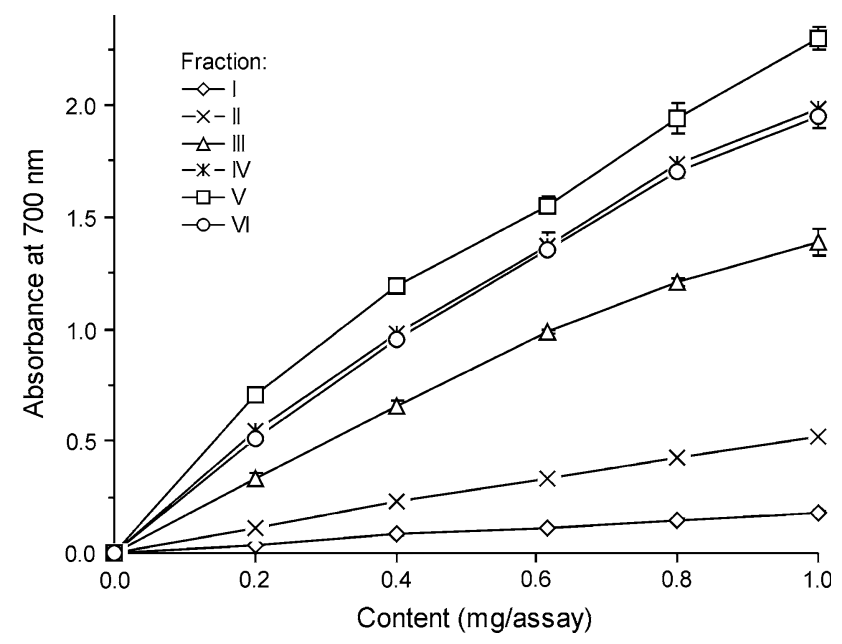

Fig. 3 Reducing power of sunflower seed fractions

which similarly to caffeic acid contains two $-\mathrm{OH}$ groups bound to an aromatic ring in the ortho position. It is known that a catechol group in flavonoid and phenolic acid structures is essential for their free radical-scavenging and metal chelating activities [33]. Consequently, it is not surprising that dicaffeoylquinic acids, containing two catechol groups in the structure, are characterized by higher antioxidant activity than that of chlorogenic acid [31, 34]. On the other hand, Kim et al. [34] compared antioxidant activity of five various dicaffeoylquinic acid derivatives that have non-esterified - $\mathrm{OH}$ groups in catechol structures. These compounds were characterized by similar (not statistically significant differences) DPPHscavenging activity and inhibition of formation of cholesteryl ester hydroperoxide during copper ion-induced rat blood plasma oxidation. Therefore, the activity of those compounds is not connected with the presence or absence of the olefinic double bond or with the binding position of caffeic and dihydrocaffeic acids to the quinic acid [34]. In our study, dicaffeoylquinic acid isomers were present mainly in fraction VI (Table 4), and it seems that those compounds mainly determined the high antioxidant activity and reducing power of this fraction (Table 5; Figs. 2, 3). The second group of compounds abundant in fraction VI was caffeoyl-dimethoxycinnamoylquinic acid isomers (Table 4). To the best of our knowledge, the antioxidant activity of those compounds has not been studied so far. It seems that due to esterification of $-\mathrm{OH}$ groups in the catechol structure, they might exert lower antioxidant effect than caffeoyl-dimethoxycinnamoylquinic acid isomers. On the other hand, high correlations between the sum of phenolic compounds in fractions and the results of antioxidant tests (Table 2) might be indicative of possible high antiradical activity and reducing power of caffeoyl-dimethoxycinnamoylquinic acid isomers. 


\section{Conclusions}

In the presented study, we fractionated crude extract of sunflower seeds using Sephadex LH-20 column chromatography with methanol as a mobile phase. This step enabled us to identify and quantify phenolic compounds that were not noted before in sunflower seeds. Until now, researchers identified non-esterified phenolic acids, isomers of caffeoylquinic acid, $p$-coumaroylquinic acid and dicaffeoylquinic acids in sunflower seeds [8-10, 12, 13]. In our research, we additionally found new isomers of coumaroylquinic acid (probably 3-O-pcoumaroylquinic and 4-O-p-coumaroylquinic acids) and dicaffeoylquinic acids (probably 1,3-di- $O$-caffeoylquinic and 1,4-di- $O$-caffeoylquinic acids). Moreover, caffeoyl-dimethoxycinnamoylquinic acid isomers and few compounds belonging to flavonoids were tentatively identified.

The results of ABTS, DPPH and $\mathrm{Fe}^{3+} /$ ferricyanide assays showed good correlations between each other and with phenolic content of individual fraction of sunflower seeds. The determination of antiradical activity and reducing power of individual fractions, containing different types of phenolic compounds, enables the conclusion that not only predominant 5-O-caffeoylquinic acid contributes to the high antioxidant activity of sunflower seeds, but also isomers of dicaffeoylquinic acid and caffeoyl-dimethoxycinnamoylquinic acid. It seems that this information can be useful for utilization of sunflower cake, by-product of oil extraction, as functional food component or as antioxidant supplement.

Conflict of interest The authors declare that they have no conflict of interest.

Open Access This article is distributed under the terms of the Creative Commons Attribution License which permits any use, distribution, and reproduction in any medium, provided the original author(s) and the source are credited.

\section{References}

1. StAngelo AJ (1996) Lipid oxidation in foods. Crit Rev Food Sci 36:175-224

2. Temple NJ (2000) Antioxidants and disease: more questions than answers. Nutr Res 20:449-459

3. Velioglu YS, Mazza G, Gao L, Oomah BD (1998) Antioxidant activity and total phenolics in selected fruits, vegetables, and grain products. J Agric Food Chem 46:4113-4117

4. Halvorsen BL, Holte K, Myhrstad MCW, Barikmo I, Hvattum E, Remberg SF, Wold AB, Haffner K, Baugerod H, Andersen LF, Moskaug JO, Jacobs DR, Blomhoff R (2002) A systematic screening of total antioxidants in dietary plants. J Nutr 132:461-471

5. Schmidt S, Pokorny J (2005) Potential application of oilseeds as source of antioxidants for food lipids - a review. Czech J Food Sci 23:93-102

6. De Leonardis A, Macciola V, Di Domenico N (2005) A first pilot study to produce a food antioxidant from sunflower seed shells (Helianthus annuus). Eur J Lipid Sci Technol 107:220-227
7. Szydłowska-Czerniak A, Trokowski K, Szłyk E (2011) Optimization of extraction conditions of antioxidants from sunflower shells (Helianthus annuus L.) before and after enzymatic treatment. Ind Crop Prod 33:123-131

8. Pedrosa MM, Muzquiz M, García-Vallejo C, Burbano C, Cuadrado C, Ayet G, Robredo LM (2000) Determination of caffeic and chlorogenic acids and their derivatives in different sunflower seeds. J Sci Food Agric 80:459-464

9. Sabir MA, Sosulski FW, Kernan JA (1974) Phenolic constituents in sunflower flour. J Agric Food Chem 22:572-574

10. Weisz GM, Kammerer DR, Carle R (2009) Identification and quantification of phenolic compounds from sunflower (Helianthus annuus L.) kernels and shells by HPLC-DAD/ESI-MS ${ }^{\mathrm{n}}$. Food Chem 115:758-765

11. Gonzalez-Perez S, Vereijken JM (2007) Sunflower proteins: overview of their physicochemical, structural and functional properties. J Sci Food Agric 87:2173-2191

12. Aramendia MA, Garcia IM, Lafont F, Lizaso J, Marinas JM, Urbano FJ (2000) Rapid determination of chlorogenic acid and related compounds in sunflower seeds by high-performance liquid chromatography/atmospheric pressure chemical ionization mass spectrometry. Rapid Commun Mass Sp 14:1019-1022

13. Dąbrowski KJ, Sosulski FW (1984) Quantitation of free and hydrolyzable phenolic-acids in seeds by capillary gas-liquidchromatography. J Agric Food Chem 32:123-127

14. Naczk M, Shahidi F (1989) The effect of methanol-ammoniawater treatment on the content of phenolic acids of canola. Food Chem 31:159-164

15. Yen GC, Chen HY (1995) Antioxidant activity of various tea extracts in relation to their antimutagenicity. J Agric Food Chem 43:27-32

16. Re R, Pellegrini N, Proteggente A, Pannala A, Yang M, RiceEvans C (1999) Antioxidant activity applying an improved ABTS radical cation decolorization assay. Free Radic Biol Med 26:1231-1237

17. Oyaizu M (1986) Studies on products of browning reaction: antioxidative activities of products of browning reaction prepared from glucosamine. Jpn J Nutr 44:307-315

18. Wanasundara U, Amarowicz R, Shahidi F (1994) Isolation and identification of an antioxidative component in canola-meal. J Agric Food Chem 42:1285-1290

19. Amarowicz R, Karamać M, Weidner S (2001) Antioxidant activity of phenolic fractions of pea (Pisum sativum). Czech $\mathrm{J}$ Food Sci 19:139-142

20. Amarowicz R, Karamać M, Shahidi F (2003) Antioxidant activity of phenolic fractions of lentil (Lens culinaris). J Food Lipids 10:1-10

21. Arfan M, Amin H, Kosińska A, Karamać M, Amarowicz R (2008) Antioxidant activity of phenolic fractions of Litsea monopetala (Persimon-leaved Litsea) bark extract. Pol J Food Nutr Sci 58:229-233

22. Amarowicz R, Karamać M, Kmita-Głażewska H, Troszyńska A, Kozłowska H (1996) Antioxidant activity of phenolic fractions of everlasting pea, faba bean and broad bean. J Food Lipids 3:199211

23. Dueñas M, Fernández ML, Hernández T, Estrella I, Muñoz R (2005) Bioactive phenolic compounds of cowpeas (Vigna sinensis L.). Modifications by fermentation with natural microflora and with Lactobacillus plantarum ATCC14917. J Sci Food Agric 85:297-304

24. Clifford MN, Johnston KL, Knight S, Kuhnert N (2003) Hierarchical scheme for $\mathrm{LC}^{-M S^{\mathrm{n}}}$ identification of chlorogenic acids. J Agric Food Chem 51:2900-2901

25. Clifford MN, Knight S, Kuhnert N (2005) Discriminating between the six isomers of dicaffeoylquinic acid by LC-MS ${ }^{\mathrm{n}}$. J Agric Food Chem 53:3821-3832 
26. Clifford MN, Knight S, Surucu B, Kunert N (2006) Characterization by LC-MS ${ }^{\mathrm{n}}$ of four new classes of chlorogenic acids in green coffee beans: dimethoxycinnamoylquinic acids, diferuloylquinic acids, caffeoyl-dimethoxycinnamoylquinic acids, and feruloyl-dimethoxycinnamoylquinic acids. J Agric Food Chem 54:1957-1969

27. Bunzel M, Ralph J, Brüning P, Steinhart H (2006) Structural identification of dehydrotriferulic and dehydrotetraferulic acids isolated from insoluble maize bran fiber. J Agric Food Chem 54:6409-6418

28. Dueñas M, Mingo-Chornet H, Pérez-Alonso JJ, Di Paola-Naranjo R, González-Paramás AM, Santos-Buelga C (2008) Preparation of quercetin glucuronides and characterization by HPLC-DADESI/MS. Eur Food Res Technol 227:1069-1076

29. Shahidi F, Chandrasekara A (2010) Hydroxycinnamates and their in vitro and in vivo antioxidant activities. Phytochem Rev 9SI: $147-170$
30. Chen JH, Ho CT (1997) Antioxidant activities of caffeic acid and its related hydroxycinnamic acid compounds. J Agric Food Chem 45:2374-2378

31. Pellati F, Benvenuti S, Magro L, Melegari M, Soragni F (2004) Analysis of phenolic compounds and radical scavenging activity of Echinacea spp. J Pharm Biomed 35:289-301

32. Karamać M, Kosińska A, Pegg RB (2005) Comparison of radicalscavenging activities for selected phenolic acids. Pol J Food Nutr Sci 55:165-170

33. Rice-Evans CA, Miller NJ, Paganga G (1996) Structure-antioxidant activity relationships of flavonoids and phenolic acids. Free Radic Biol Med 20:933-956

34. Kim JY, Cho JY, Ma YK, Park KY, Lee SH, Ham KS, Lee HJ, Park KH, Moon JH (2011) Dicaffeoylquinic acid derivatives and flavonoid glucosides from glasswort (Salicornia herbacea L.) and their antioxidative activity. Food Chem 125:55-62 